\title{
Effet de la mise en culture des forêts secondaires sur les peuplements de macroinvertébrés du sol dans la zone de contact forêt-savane de Côte d'Ivoire
}

\author{
Jérôme Ebagnerin TONDOH \\ UFR des Sciences de la Nature / Centre de Recherche en Ecologie \\ Université d'Abobo-Adjamé, 02 BP 801 Abidjan 02, Côte d'Ivoire \\ E-mail : tondoh@uabobo.ci, tondohj@yahoo.fr \\ Reçu le 15-06-2006, accepté le 12-11-2006.
}

\begin{abstract}
Résumé
L'impact de la mise en culture des forêts sur les peuplements de macroinvertébrés du sol a été étudié en moyenne Côte d'Ivoire avec pour objectif d'identifier les plus sensibles à la perturbation des forêts. Les macroinvertébrés du sol de parcelles de forêt secondaire et de cultures vivrières ont été échantillonnés en utilisant la méthode TSBF. A l'exception de la jachère, la mise en culture de la forêt secondaire a entraîné une réduction de la densité de $76,2 \%, 22,1 \%, 22,1$ $\%, 0,92 \%$, respectivement dans les champs de maïs, d'arachide, de riz et de bananiers plantain. Une Analyse en Composantes Principales (ACP) a montré que le «type de culture» et «l'effet du feu» sont susceptibles d'être les principaux facteurs qui déterminent l'abondance et la distribution des macroinvertébrés. Par ailleurs, les Fourmis, les Diplopodes, les Vers de terre, et les Coléoptères, se sont avérés comme les macroinvertébrés les plus sensibles à la dégradation des forêts.
\end{abstract}

Mots clés: Macroinvertébrés du sol, forêts, cultures vivrières, bio-indicateurs

\begin{abstract}
Impact of cropping systems in secondary forest areas on soil macro-invertebrate communities in the buffer zone forest-savanna of Côte d'Ivoire.

The impact of cropping systems in the secondary forest area on soil macro-invertebrates was investigated in middle Côte d'Ivoire with the aim of identifying the most sensitive organisms to forest perturbation. Soil macro-invertebrates from different cropping systems were sampled using TSBF methodology and their abundance were compared to the secondary forest referred as control. Except fallow, the conversion of secondary forest has reduced the density of macro-invertebrates at $76.2 \%, 22.1 \%, 22.1 \%, 0.92 \%$, under maize, groundnut, rice and plantain banana fields respectively. A Principal Component Analysis (PCA) showed that the type of land-use and fire are factors controlling abundance and distribution of soil macro-invertebrates. In addition, ants, diplopoda, earthworms and coleoptera are likely to be the most sensitive organisms to forest perturbation.
\end{abstract}

Key words: Soil macro-invertebrates, forests, food crops, bio-indicators 


\section{Introduction}

La culture itinérante sur brûlis demeure une pratique agricole très répandue dans les pays tropicaux en général et en Afrique subsaharienne en particulier, malgré les conséquences négatives qui en résultent: déforestation, invasion des adventices, érosion et baisse de la fertilité des sols (Devendra \& Thomas, 2002). En Côte d'Ivoire, les études concernant l'impact de cette pratique sur les sols ont davantage concerné les modifications morphologiques et l'évolution des paramètres physico-chimiques (Moreau, 1983 ; Tamia, 1997). Malgré le rôle actif qui leur est attribué dans le fonctionnement du sol (Brussaard et al., 1997; Lavelle, 1997) et leur utilisation potentielle comme indicateur de la qualité des sols (Decaëns et al., 1994 ; Barros et al., 2002), les organismes du sol ont été insuffisamment étudiés en Côte d'Ivoire. Les nématodes phytoparasites ont fait l'objet d'études dans les régions forestières de Taï et d'Oumé (Fortuner \& Couturier, 1983 ; Adiko, 1988 ; Gnonhouri \& Adiko, 2000) à cause des dégâts de leurs peuplements sur les productions agricoles. Les myriapodes diplopodes et les macroinvertébrés en général ont néanmoins fait l'objet d'études comparées de leurs peuplements en milieux naturels (savane, forêt) et perturbés (cultures pérennes et vivrières) (Aouti, 1978 ; Kouassi, 1987 ; 1999). Récemment, la sensibilité des peuplements de termites et de fourmis à l'exploitation forestière a été démontrée dans la Forêt Classée de la Haute Dodo (Konaté et al., 2005). Plus globalement, les peuplements de macroinvertébrés ont été étudiés dans leur ensemble, sous une succession de plantations d'Hévéa d'âge variant de 5 à 25 ans, en comparaison avec la forêt d'origine (Gilot et al., 1995).

L'objectif de ce travail est d'étudier l'effet de la mise en culture des forêts semi-décidues de la zone de contact forêt-savane, sur les peuplements de macroinvertébrés du sol. Cette zone est, en effet, caractérisée par une prépondérance de cultures vivrières parce que les sols semblent inappropriés aux cultures de caféiers et de cacaoyers. La présente étude permettra d'identifier les groupes de macroinvertébrés les plus sensibles à la dégradation des forêts, afin de pouvoir les utiliser comme outils de suivi pour la conservation des rares écosystèmes forestiers de cette région.

\section{Matériel et méthodes}

\subsection{Site d'étude}

Cette étude s'est déroulée dans la zone de contact forêt-savane en moyenne Côte d'Ivoire, plus précisément dans la zone tampon de la Réserve de Lamto $\left(5^{\circ} 02^{\prime} \mathrm{W}, 6^{\circ} 13^{\prime} \mathrm{N}\right)$.

Le paysage végétal est caractérisé par une diversité forestière due aux activités humaines passées et récentes : mise en cultures et feu de brousse (Devineau, 1975). Les relevés floristiques ont permis de classer les forêts semidécidues de la zone d'étude dans la catégorie de forêts secondaires jeunes dont la mise en culture remonte à une vingtaine d'années (Devineau, 1984).

La pluviométrie de l'année 1992 de la zone d'étude était de $989,5 \mathrm{~mm}$ avec un maximum (224,2 $\mathrm{mm}$ ) dans le mois d'Avril. Elle est inférieure de $10 \%$ à la moyenne des 29 dernières années qui oscille autour de $1100 \mathrm{~mm}$. Les températures moyennes sous abris varient entre $26^{\circ} \mathrm{C}$ (juillet) et $30,1^{\circ} \mathrm{C}$ (mars).

Les sols sont de type ferralitique et caractérisés par un horizon superficiel très sableux surmontant une nappe gravillonnaire (Riou, 1974).

\subsection{Parcelles d'étude}

Les parcelles d'étude sont regroupées en trois catégories : les milieux naturels (forêt secondaire intacte et dégradée), les cultures vivrières (maïs, arachide, banane plantain, riz) et une jachère. L'histoire culturale des parcelles d'étude a été reconstituée après enquête auprès des paysans. Compte tenu de l'extrême dégradation des forêts secondaires, on a émis l'hypothèse selon laquelle la forêt secondaire intacte représente la parcelle témoin. La forêt secondaire dégradée a été brûlée accidentellement par les feux de brousse en 1984, pratique courante dans la région. Elle est caractérisée par une dominance de la strate herbacée en Chromolaena odorata (Asteraceae), adventice néotropicale indicatrice de la perturbation des forêts. Les parcelles de maïs, d'arachide, et de riz sont âgées d'un an, la parcelle de bananiers plantain, de deux ans. La mise en place de ces parcelles a été réalisée après abattage et brûlis des arbres et arbustes en fin de saison sèche (février-mars). Le feu est donc le principal agent perturbateur car le labour du sol est inexistant et se résume à un sarclage 
qui se caractérise par un léger travail du sol. La jachère à $C$. odorata consécutive à une culture d'igname, est âgée de 10 ans.

L'analyse des caractéristiques physiques et chimiques des sols de forêts secondaire et de la parcelle de maïs (Tableau 1 ), a révélé que la texture est sableuse avec des proportions de sable de 85 et $86,3 \%$ respectivement à $0-10$ et 10-20 cm. La conversion des forêts en culture de maïs a entraîné, d'une part, une réduction de la teneur en carbone organique $(-19,4 \%)$ et azote total $(-20,4 \%)$ dans les 10 premiers $\mathrm{cm}$ du sol, et l'acidification du sol d'autre part.

Tableau 1: Caractéristiques physico-chimiques des 20 premiers $\mathrm{cm}$ du sol des parcelles de forêt secondaire et de maïs.

\begin{tabular}{lcccc}
\hline & \multicolumn{2}{c}{ Forêt secondaire } & \multicolumn{2}{c}{ Maïs } \\
\hline & $0-10 \mathrm{~cm}$ & $10-20 \mathrm{~cm}$ & $0-10 \mathrm{~cm}$ & $10-20 \mathrm{~cm}$ \\
Sable (\%) & 85 & 86,3 & 83 & 87 \\
Limon (\%) & 10,5 & 11,3 & 9,1 & 10,2 \\
Argile (\%) & 4,5 & 2,4 & 3,2 & 1,9 \\
$\mathrm{C}(\%$ o) & 12,6 & 5,6 & 10,2 & 5,1 \\
$\mathrm{~N}(\%$ o) & 1,3 & 0,5 & 1 & 00,52 \\
$\mathrm{pH}_{\text {eau }}$ & 7,5 & 6,8 & 6,9 & 5,8 \\
\hline
\end{tabular}

\subsection{Echantillonnage des macroinvertébrés du sol}

Les macroinvertébrés, organismes du sol dont la taille est supérieure à $2 \mathrm{~mm}$ ont été échantillonnés dans la période de juin à septembre 1992. Dans chaque parcelle d'étude, ces organismes ont été prélevés dans des monolithes de sol (25x25x30 $\mathrm{cm}$ ) juxtaposés tous les $5 \mathrm{~m}$, prélevés le long d'un transect (Lavelle \& Pashanasi, 1989) et triés manuellement. Cette méthode connue sous l'appellation «méthode TSBF» est recommandée par le Programme TSBF (Tropical Soil Biology and Fertility) pour comparer l'abondance des macroinvertébrés du sol dans les écosystèmes naturels et anthropisés.

Cinq à dix monolithes ont été prélevés par parcelle d'étude selon leur superficie. Les macroinvertébrés du sol sont ensuite récoltés par tri manuel direct par tranches de $10 \mathrm{~cm}$ de sol et conservés dans du formol à $4 \%$ (vers de terre) ou de l'alcool à 70 $\%$ (autres animaux). L'identification par groupes taxonomiques, le comptage et la pesée des individus ont été réalisés au laboratoire.

\subsection{Analyses statistiques}

Une analyse en composantes principales (ACP) a été réalisée sur les données obtenues afin d'expliquer l'impact des pratiques culturales sur l'abondance du peuplement de macroinvertébrés du sol. Le module "PCA» du logiciel ADE-4 (Thioulouse et al., 1997) a été utilisé à cet effet.

\section{Résultats}

\subsection{Densité des peuplements de macro- invertébrés}

La densité globale moyenne des macroinvertébrés du sol varie selon les types d'utilisation (Tableau 2). La jachère à $C$. odorata héberge la plus forte densité $\left(6540,8 \pm 4509,7\right.$ ind $\left.\cdot \mathrm{m}^{-2}\right)$. Elle est suivie de la forêt secondaire $\left(3308,8 \pm 1630\right.$ ind. $\left.\mathrm{m}^{-2}\right)$ et de la bananeraie $\left(3278,4 \pm 1695,1\right.$ ind. $\left.\mathrm{m}^{-2}\right)$. La plus faible densité $\left(784,8 \pm 249,7\right.$ ind. $\left.\mathrm{m}^{-2}\right)$ a été observée dans le champ de maïs. Les principaux groupes taxonomiques sont les termites $(227,2$ \pm 114 - 5609, $6 \pm 4433,2$ ind. $\mathrm{m}^{-2}$ ), les fourmis $\left(302 \pm 184-1179,8 \pm 1456,7\right.$ ind. $\left.\mathrm{m}^{-2}\right)$ et les vers de terre $\left(39,2 \pm 39,2-235,2 \pm 40,7\right.$ ind. $\left.\mathrm{m}^{-2}\right)$ sur l'ensemble des milieux échantillonnés. Ces groupes représentent l'essentiel du peuplement, leurs proportions atteignent 89,3 et $66,7 \%$, respectivement dans la bananeraie et dans la rizière.

La densité de termites est plus importante dans la jachère à $C$. odorata $\left(5609,6 \pm 4433,2\right.$ ind. $\left.\mathrm{m}^{-2}\right)$ et dans la bananeraie $\left(2928 \pm 1704,6\right.$ ind. $\left.\mathrm{m}^{-2}\right)$. Celle des fourmis a les plus fortes valeurs dans les champs de riz (1672 $\pm 1710,2$ ind. $\left.\mathrm{m}^{-2}\right)$ et d'arachide $\left(1537,6 \pm 1226\right.$ ind. $\left.\mathrm{m}^{-2}\right)$. 
Tableau 2 : Densité moyenne (ind. $\mathrm{m}^{-2} \pm$ erreur standard) des macroinvertébrés du sol dans 6 types d'utilisation de sol

\begin{tabular}{|c|c|c|c|c|c|c|c|}
\hline \multicolumn{8}{|c|}{ Forêt secondaire } \\
\hline & Forêt secondaire & brûlée & Jachère & Maïs & Arachide & Riz & Banane plantain \\
\hline Vers de terre & $235,2 \pm 40,7$ & $150,4 \pm 33,7$ & $92,8 \pm 26,7$ & $39,2 \pm 39,2$ & $68,8 \pm 17,8$ & $185,6 \pm 43,4$ & $185,6 \pm 31,6$ \\
\hline Termites & $990,4 \pm 384,4$ & $408 \pm 351,2$ & $5609,6 \pm 4433,2$ & $227,2 \pm 114$ & $843,2 \pm 810,3$ & $574,4 \pm 320,9$ & $2928 \pm 1704,6$ \\
\hline Fourmis & $1179,8 \pm 1456,7$ & $302 \pm 184$ & $678,4 \pm 220,6$ & $423,2 \pm 229,7$ & $1537,6 \pm 1226$ & $1672 \pm 1710,2$ & $100,8 \pm 77,6$ \\
\hline Coléoptères & $65,6 \pm 14,8$ & $44,8 \pm 8,6$ & $35,2 \pm 6,7$ & $26,4 \pm 6,3$ & $44,8 \pm 13$ & $25,6 \pm 8$ & $20,8 \pm 11$ \\
\hline Dermaptères & $27,2 \pm 11,3$ & $75,2 \pm 10,7$ & $28,8 \pm 8,8$ & $7,2 \pm 2,5$ & $22,4 \pm 6,7$ & $19,2 \pm 8,3$ & $6,4 \pm 3,7$ \\
\hline Hémiptères & $3,2 \pm 2,2$ & $1,6 \pm 1,7$ & 0 & 0 & $8 \pm 3,8$ & 0 & 0 \\
\hline \multicolumn{8}{|l|}{ Lépidoptères } \\
\hline (larves) & 0 & $6,4 \pm 4,5$ & 0 & 0 & 0 & $3,2 \pm 3,4$ & 0 \\
\hline Arachnides & $12,8 \pm 6,1$ & $49,6 \pm 15,6$ & $28,8 \pm 13,3$ & $14,4 \pm 4,9$ & $12,8 \pm 5,5$ & $14,4 \pm 7,3$ & $6,4 \pm 2,8$ \\
\hline Diplopodes & $92,8 \pm 18,6$ & $59,2 \pm 12,8$ & $38,4 \pm 25,7$ & $14,4 \pm 4,6$ & $11,2 \pm 5,6$ & $3,2 \pm 2,2$ & $6,4 \pm 3,7$ \\
\hline Chilopodes & $41,6 \pm 10,7$ & $152 \pm 25,8$ & $25,6 \pm 12,1$ & $26,4 \pm 7,1$ & $20,8 \pm 6,7$ & $6,4 \pm 5,2$ & $19,2 \pm 10,3$ \\
\hline Isopodes & $30,4 \pm 15,6$ & 0 & $3,2 \pm 3,6$ & $6,4 \pm 2,2$ & 0 & 0 & 0 \\
\hline Gastéropodes & $4,8 \pm 3,6$ & $1,6 \pm 1,7$ & 0 & 0 & 0 & 0 & 0 \\
\hline Divers & $8 \pm 3,8$ & $4,8 \pm 3,6$ & 0 & 0 & $8 \pm 2,8$ & $1,6 \pm 1,7$ & $4,8 \pm 3,6$ \\
\hline Total & $3308,8 \pm 1630$ & $1256 \pm 404,8$ & $6540,8 \pm 4509,7$ & $784,8 \pm 249,7$ & $2577,6 \pm 1444,5$ & $2505,6 \pm 1713,8$ & $3278,4 \pm 1695,1$ \\
\hline
\end{tabular}

Le cercle de corrélation de l'Analyse en Composantes Principales (ACP) a révélé qu'à l'exception des termites, tous les groupes taxonomiques sont négativement corrélés à l'axe 1 qui représente $38,8 \%$ de l'inertie totale (Fig. 1a). La position de la variable termite le long de l'axe 1 de l'ACP peut s'expliquer par le fait que sa densité est particulièrement élevée sous la jachère à $C$. odorata. Par ailleurs, l'analyse du cercle de corrélation a montré que le peuplement de macroinvertébrés est séparé en trois principaux groupes : le premier groupe comporte les termites, le second groupe est constitué des diplopodes, vers de terre, coléoptères, gastéropodes, hémiptères, isopodes, caractérisés par une réduction de leur densité par la dégradation et la mise en culture de la forêt secondaire. Le troisième groupe concerne l'ensemble formé par les chilopodes, dermaptères, arachnides, lépidoptères, dont les densités se caractérisent, soit par une augmentation, soit par une réduction. L'axe 2 $(27,9 \%$ de l'inertie totale) montre que le peuplement en macroinvertébrés varie en fonction du type d'utilisation du sol, car il est positivement ou négativement corrélé aux groupes taxonomiques. Les fourmis, à un degré moindre, les hémiptères, les isopodes et les vers de terre sont négativement corrélés à l'axe 2, révélant ainsi leur sensibilité à la perturbation des forêts par le feu.

La projection des types d'utilisation du sol dans le plan factoriel 1-2 (Fig. 1b) a permis de révéler l'effet de deux types de facteurs. L'axe 1 qui oppose les milieux naturels aux milieux cultivés traduit l'effet du "type d'utilisation du sol» sur les peuplements de macroinvertébrés du sol tandis que l'axe 2 oppose principalement les deux types de forêts secondaires qui se distinguent par l'effet du feu. 


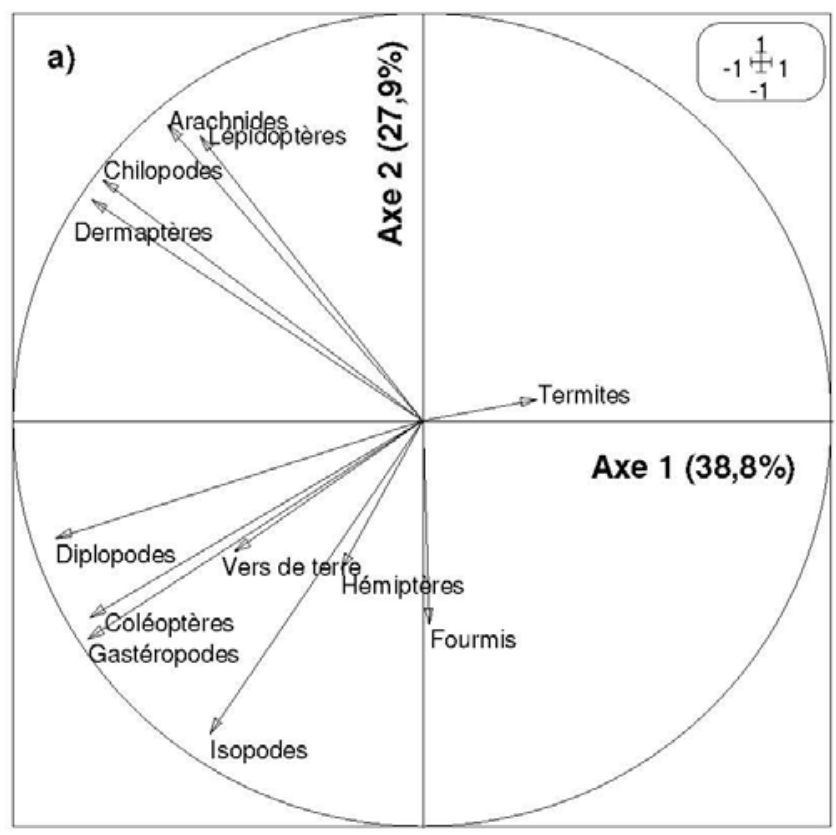

Figure 1a : Cercle de corrélation de l'ACP montrant les relations entre les groupes taxonomiques du peuplement de macroinvertébrés du sol

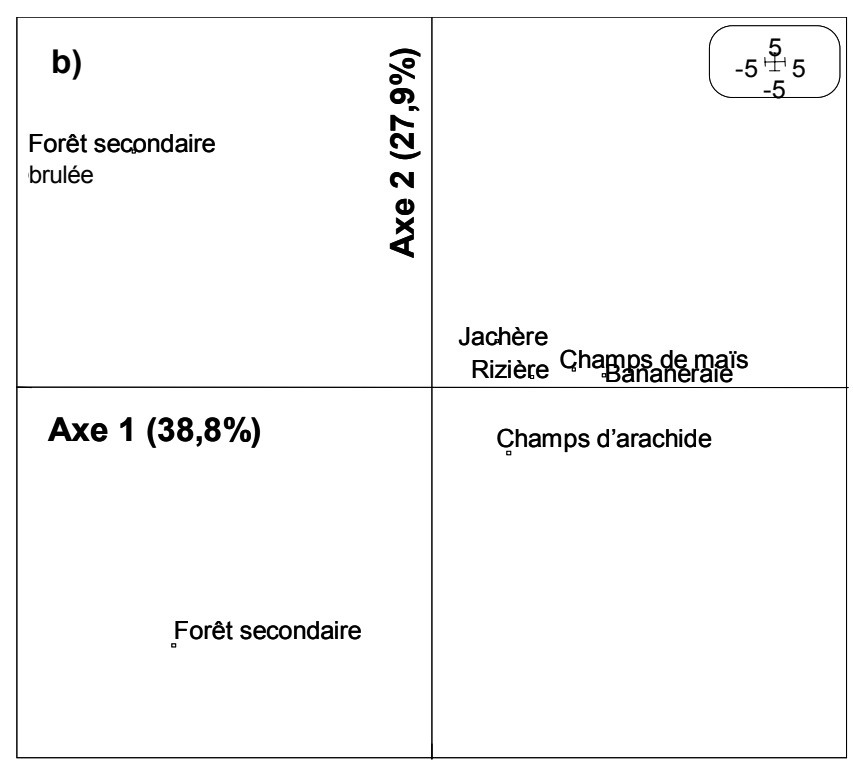

Figure 1b : Projection des types d'utilisation du sol dans le plan factoriel 1-2 


\subsection{Biomasse des macroinvertébrés}

La biomasse globale du peuplement de macroinvertébrés est caractérisée par d'importantes fluctuations (Tableau 3). Les plus fortes valeurs ont été obtenues dans la rizière $\left(35,4 \pm 26,8 \mathrm{~g} \cdot \mathrm{m}^{-2}\right)$ et la forêt secondaire $(23,7 \pm$ $\left.7,7 \mathrm{~g} \cdot \mathrm{m}^{-2}\right)$. La jachère à $C$. odorata $\left(19 \pm 9,1\right.$ ind. $\mathrm{m}^{-}$ ${ }^{2}$ ) et la bananeraie $\left(14,1 \pm 3,1 \mathrm{~g} \cdot \mathrm{m}^{-2}\right)$ hébergent des peuplements à valeurs intermédiaires, alors que la forêt secondaire dégradée $\left(9,8 \pm 2,5 \mathrm{~g} \cdot \mathrm{m}^{-}\right.$ $\left.{ }^{2}\right)$, les champs d'arachide $\left(8,8 \pm 3,1 \mathrm{~g} \cdot \mathrm{m}^{-2}\right)$ et de maïs $\left(5,1 \pm 1,3{\left.\mathrm{~g} \cdot \mathrm{m}^{-2}\right)}^{2}\right.$ affichent les plus faibles valeurs de biomasse. Seuls les diplopodes ont montré une tendance à la réduction de leur biomasse, en réponse à la dégradation et à la mise en culture de la forêt secondaire. La perte de biomasse est de 52,3\%, 89,3\%; $93.5 \%, 91,7$ $\%$; $99,8 \%$; et $99,3 \%$ respectivement dans la forêt secondaire dégradée, la jachère, le champ de maïs, le champ d'arachide, la rizière et la bananeraie. Les fourmis, les vers de terre, les termites et les diplopodes représentent les plus importants groupes car leurs proportions peuvent atteindre respectivement 70,7 \%; 60,1 \%; 55,7 \% et $41,8 \%$ dans la rizière, la bananeraie, la jachère et la forêt secondaire dégradée.

Tableau 3 : Biomasse moyenne ( $\mathrm{g} \mathrm{m}^{-2} \pm$ erreur standard) des macroinvertébrés du sol dans les types d'utilisation de sol

\begin{tabular}{|c|c|c|c|c|c|c|c|}
\hline & $\begin{array}{c}\text { Forêt } \\
\text { secondaire }\end{array}$ & $\begin{array}{c}\text { Forêt } \\
\text { secondaire } \\
\text { Brulée }\end{array}$ & Jachère & Maïs & Arachide & Riz & $\begin{array}{l}\text { Banane } \\
\text { plantain }\end{array}$ \\
\hline Vers de terre & $5,32 \pm 1,19$ & $3,06 \pm 0,82$ & $3,7 \pm 1,87$ & $2,92 \pm 1,3$ & $2,43 \pm 1,11$ & $8,11 \pm 3,95$ & $8,45 \pm 1,98$ \\
\hline Termites & $2,76 \pm 1,27$ & $0,18 \pm 0,08$ & $10,57 \pm 8,23$ & $0,18 \pm 0,09$ & $1,81 \pm 1,76$ & $1,43 \pm 0,83$ & $3,84 \pm 2$ \\
\hline Fourmis & $3,92 \pm 2,54$ & $0,36 \pm 0,1$ & $3,03 \pm 1,73$ & $0,5 \pm 0,2$ & $2,68 \pm 0,11$ & $25,01 \pm 26,12$ & $0,16 \pm 0,08$ \\
\hline Coléoptères & $1,95 \pm 0,82$ & $0,40 \pm 0,16$ & $0,37 \pm 0,29$ & $0,55 \pm 0,32$ & $0,55 \pm 0,02$ & $0,3 \pm 0,16$ & $1,11 \pm 0,79$ \\
\hline Dermaptères & $0,11 \pm 0,07$ & $0,11 \pm 0,02$ & $0,08 \pm 0,02$ & $0,02 \pm 0,01$ & $0,1 \pm 0,01$ & $0,08 \pm 0,03$ & $0,05 \pm 0,03$ \\
\hline Hémiptères & $0,14 \pm 0,1$ & $0,05 \pm 0,05$ & 0 & 0 & $0,33 \pm 0,01$ & 0 & 0 \\
\hline \multicolumn{8}{|l|}{ Lépidoptères } \\
\hline (larves) & 0 & $0,07 \pm 0,05$ & 0 & 0 & 0 & $0,19 \pm 0,2$ & 0 \\
\hline Arachnides & $0,22 \pm 0,18$ & $0,11 \pm 0,04$ & $0,22 \pm 0,19$ & $0,09 \pm 0,06$ & $0,04 \pm 0,001$ & $0,16 \pm 0,11$ & $0,16 \pm 0,11$ \\
\hline Diplopodes & $8,59 \pm 4,1$ & $4,1 \pm 2,55$ & $0,92 \pm 0,45$ & $0,56 \pm 0,27$ & $0,71 \pm 0,03$ & $0,02 \pm 0,02$ & $0,06 \pm 0,04$ \\
\hline Chilopodes & $0,2 \pm 0,06$ & $1,02 \pm 0,21$ & $0,08 \pm 0,04$ & $0,16 \pm 0,06$ & $0,14 \pm 0,01$ & $0,01 \pm 0,01$ & $0,11 \pm 0,07$ \\
\hline Isopodes & $0,16 \pm 0,13$ & 0 & $0,01 \pm 0,01$ & $0,08 \pm 0,05$ & 0 & 0 & 0 \\
\hline Gastéropodes & $0,04 \pm 0,03$ & $0,19 \pm 0,2$ & 0 & 0 & 0 & 0 & 0 \\
\hline Divers & $0,26 \pm 0,19$ & $0,16 \pm 0,17$ & 0 & 0 & $0,04 \pm 0,002$ & $0,06 \pm 0,06$ & $0,12 \pm 0,1$ \\
\hline Total & $23,7 \pm 7,7$ & $9,8 \pm 2,5$ & $19 \pm 9,1$ & $5,1 \pm 1,3$ & $8,8 \pm 3,1$ & $35,4 \pm 26,8$ & $14,10 \pm 3,1$ \\
\hline
\end{tabular}

\section{Discussion}

L'abondance des macroinvertébrés du sol des forêts secondaires semi-décidues de la zone de contact forêt-savane est de loin inférieure à celle des forêts sempervirentes de la zone humide (5750 ind $\mathrm{m}^{-2} ; 75 \mathrm{~g} \mathrm{~m}^{-2}$ ), situées dans la région de Bimbresso (Gilot et al., 1995). La pluviométrie serait l'un des facteurs déterminant la distribution et l'abondance de ces organismes. Nos résultats sont similaires à ceux obtenus par Lavelle \&
Pashanasi (1989) dans les forêts secondaires d'Amazonie péruvienne.

A l'exception de la bananeraie, la mise en culture de la forêt secondaire s'est traduite par une réduction de la densité globale des macroinvertébrés du sol. L'augmentation de la densité dans la jachère à $C$. odorata est due à l'expansion des populations de termites. La réduction de biomasse est plus marquée dans les milieux cultivés, à l'exception de la rizière où elle s'est accrue de 1,5 fois. Cette augmentation 
serait due à la pullulation des populations de fourmis et de vers de terre. D'une manière générale, les populations de macroinvertébrés sont sensibles à l'action du feu. Cette assertion est confirmée par l'importante réduction des macroinvertébrés dans la forêt secondaire brûlée où 8 ans après le passage du feu le peuplement n'a pu se reconstituer.

Les résultats de l'Analyse en Composantes Principales ont montré que l'établissement de cultures vivrières à partir des forêts secondaires perturbe considérablement les peuplements de macroinvertébrés du sol par l'intermédiaire de deux facteurs : le type de culture et l'effet du feu. Les cultures caractérisées par un microclimat humide (bananeraies, rizières) sont sujettes à une réduction sensible ou une augmentation marquée des principaux groupes taxonomiques (termites, fourmis, vers de terre). Le peuplement de termites est dominé par le groupe trophique des champignonnistes (Ancistrotermes cavithorax surtout) comme l'ont montré Wood et al. (1977) au Nigeria où les termites champignonnistes survivent dans les milieux cultivés et peuvent atteindre cinq fois leur densité initiale. En ce qui concerne les fourmis, Folgarait et al. (2002) ont également montré que la pullulation des nids de fourmis de Camponotus punctulatus dans les champs de riz irrigué du Nord-Est de l'Argentine était due à la modification de leur régime alimentaire et des conditions microclimatiques. La réduction de l'abondance des vers de terre dans les milieux dégradés par l'action du feu a été évoquée par Fragoso et al. (1997) et aurait pour cause la réduction des espèces de litière. Ces résultats confirment les observations de Lavelle \& Pashanasi (1989) selon lesquelles l'établissement d'une culture de manioc en Amazonie Péruvienne a entraîné une réduction de 84 et $57,5 \%$ de la densité et de la biomasse. La qualité des résidus végétaux d'Arachis hypogea et de C. odorata, source principale de nourriture, serait à l'origine de la recolonisation des macroinvertébrés dans la jachère et dans le champ d'arachide.

La dégradation accidentelle des forêts par le feu et l'utilisation du feu pour le défrichement de la forêt secondaire ont grandement contribué à réduire l'abondance des macroinvertébrés du sol. Aouti (1978) a montré que les myriapodes diplopodes représentaient non seulement l'essentiel du peuplement de macroinvertébrés du sol dans la forêt classée de Téké, mais étaient caractérisés par une importante réduction (-47,5 $\%$ ) de leur abondance dans les plantations d'Hévéa issues de la forêt. Les fourmis et secondairement les diplopodes et les vers de terre ont été particulièrement sensibles à l'action du feu. La destruction de la litière de la forêt secondaire par le feu serait à l'origine de la réduction de fourmis, des diplopodes et des vers de terre épigés qui vivent majoritairement dans les 20 premiers $\mathrm{cm}$ du sol (Aouti, 1978; Lavelle, 1983; Diomandé, 1984).

\section{Conclusion}

La mise en culture des forêts secondaires a eu un effet dépressif principalement sur les fourmis, les diplopodes, les vers de terre, et les coléoptères. Ces groupes taxonomiques, considérés comme les plus sensibles à la dégradation des forêts par les activités humaines, peuvent être utilisés comme des «bioindicateurs» de l'état de conservation des forêts. Par ailleurs, la reconstitution rapide des peuplements de macroinvertébrés dans la jachère à $C$. odorata et sous la bananeraie montre que l'adoption de système d'utilisation du sol susceptible de créer un micro-climat favorable à l'activité des macroinvertébrés du sol, peut contribuer à une meilleure conservation de ces organismes dont le rôle dans le fonctionnement du sol est avéré.

\section{Références citées}

Adiko A., 1988. Plant-parasitic nematodes associated with plantain, Musa paradisiaca (AAB), in Ivory Coast. Rev. Nématol. 11: 109-113.

Tamia A.J., 1997. Modifications physiques d'un sol ferralitique sous différents modes de mise en culture en Côte d'lvoire forestière: conséquence sur le développement du maïs. Thèse de Doctorat. Université Paris XII Val-deMarne, $271 \mathrm{p}$.

Aouti S., 1978. Etude comparée des peuplements de myriapodes diplopodes d'une forêt hygrophile et d'une plantation d'hévéa en basse Côte d'Ivoire. Ann. Univ. Abidjan série E, XI: 8-32.

Barros E., Pashanasi B., Constantino R. \& Lavelle P., 2002. Effects of land-use systems on the soil macrofauna in western Brazilian Amazonia. Biol. Fert. Soils 35: 338-347. 
Brussaard L., Behan-Pelletier M.V., Bignell E.D., Brown K.V., Didden W., Folgarait P., Fragoso C., Freckman W.D., Gupta V.S.R.V., Hattori T., Hawksworth L.D., Klopatek C., Lavelle P., Malloch W.D., Russek J., Sodorstom B., Tiedje M.,J. \& Virginia A.R., 1997. Biodiversity and ecosystem functioning in soil. Ambio 26: 563-570.

Decaëns T., Lavelle P., Jimenez J.J., Escobar G. \& Rippstein G., 1994. Impact of land management on soil macrofauna in the Oriental Llanos of Colombia. Eur. J. Soil Biol. 30: 157-168.

Devendra C. \& Thomas D., 2002. Smallholders farming systems in Asia. Agri. Syst. 71: 17-25.

Devineau J.L., 1975. Etudes quantitative des forêtgaleries de Lamto (Côte d'Ivoire). Thèse de Doctorat, 3è cycle. Université Paris VI, 171 pp.

Devineau J.L., 1984. Structure et dynamique de quelques forêts tropicales de l'Ouest africain (Côte d'Ivoire). Travaux des Chercheurs de Lamto, 5, $291 \mathrm{pp.}$

Diomandé T., 1984. Description de la structure du nid chez quelques fourmis terricoles et forestières. Ann. Univ. Abidjan, série E, XI: 195-224.

Folgarait P.J., Thomas F., Desjardins T., Grimaldi M., Tayasu I., Curmi P. \& Lavelle P., 2003. Soil properties and the macrofauna community in abandoned irrigated rice fields of northeastern Argentina. Biol. Fert. Soils 38: 349-357.

Fortuner R. \& Couturier G., 1983. Les nématodes parasites de plantes de la forêt de Taï (Côte d'Ivoire). Rev. Nématol. 6: 3-10.

Fragoso C., Brown G.G., Patron J.C., Blanchart E., Lavelle P., Pashanasi B., Senapati B. \& Kumar T., 1997. Agricultural intensification, soil biodiversity and agroecosystem function in the tropics: the role of earthworms. Appl. Soil Ecol. 6: 17-35.

Gilot C., Lavelle P., Blanchart E., Keli J., Kouassi P. \& Guillaume G., 1995. Biological activity of soil under rubber plantations in Côte d'Ivoire. Acta Zool. Fennica 196: 186-189.

Gnonhouri G.P. \& Adiko A., 2000. Effet des cultures après jachère améliorée sur les populations de nématode en Côte d'Ivoire. In : Foret C. \& Pontanier R., Eds. La jachère en Afrique tropicale. Rôles, Aménagement, Alternatives. Actes du Séminaire international (Volume 1). 13-16 avril 1999, Dakar
Sénégal : John Libbey Eurotext, Paris, France, pp. 277-282.

Konaté S., Yéo K., Yoboué L., Alonso E.L. \& Kouassi K., 2005. Evaluation rapide de la diversité des insectes des Forêts Classées de la Haute Dodo et du Cavally, Côte d'Ivoire. In : Alonso E.L., Lauginie F. \& Rondeau, G. Eds. Une évaluation biologique de deux forêts classes du sud-ouest de la Côte d'Ivoire. Bulletin RAP d'évaluation rapide $N^{\circ} 34$, Conservation International, Washington D.C., pp. 39-49.

Kouassi K.P., 1987. Etude comparative de la macrofaune endogé d'écosystème guinéens naturels et transformés de Côte d'lvoire. Thèse de Doctorat, 3è cycle. Université Nationale de Côte d'Ivoire, 129 pp.

Kouassi K.P., 1999. Structure et dynamique des groupes trophiques de la macrofaune du sol d'écosystèmes naturels et transformés de Côte d'Ivoire. Thèse de Doctorat d'Etat ès Sciences Naturelles. Université de Cocody, 206 pp.

Lavelle P., 1983. The structure of earthworm communities. In: Satchell, J.E. Ed. Earthworm ecology: From Darwin to Vermiculture. Chapman and Hall, London, pp. 449-466.

Lavelle P., 1997. Faunal activities and soil processes: adaptive strategies that determine ecosystem function. Adv. Ecol. Res. 27: 93-132.

Lavelle P. \& Pashanasi, B., 1989. Soil macrofauna and land management in Peruvian Amazonia (Yurimaguas, Loreto). Pedobiologia 33: 283-291.

Moreau R., 1983. Evolution des sols sous différents modes de mise en culture, en Côte d'Ivoire forestière et préforestière. Cah. O.R.T.O.M., sér. Pédol. XX : 311-325.

Riou G., 1974. Les sols de la savane de Lamto. In : Analyse d'un écosystème tropical humide : la savane de Lamto (Côte d'Ivoire). Bulletin de Liaison des Chercheurs de Lamto. Les facteurs physiques du milieu 1: 3-45.

Thioulouse J., Chessel D., Dolédec S. \& Olivier J.M., 1997. ADE-4: a multivariate analysis and graphical display software. Stat. Comput. 7: 75-83.

Wood T.G., Johnson R.A. \& Ohiagu C.E., 1977. Populations of termites (Isoptera) in natural and agricultural ecosystems in Southern Guinea savanna near Mokwa, Nigeria. Geo-Eco-Trop 1: 139-148. 